\title{
Tramadol use in a patient with Brugada syndrome and morphine allergy: a case report
}

This article was published in the following Dove Press journal: Journal of Pain Research

\author{
Cengiz Sahutoglu \\ Seden Kocabas \\ Fatma Zekiye Askar \\ Ege University School of Medicine, \\ Department of Anesthesiology and \\ Reanimation, Izmir, Turkey
}

\begin{abstract}
Brugada syndrome is a rare syndrome characterized by arrhythmias and sudden death, particularly in younger individuals. A mutation in a gene encoding the human cardiac sodium channels is responsible for this syndrome. In the literature, there are several case reports of Brugada syndrome in association with the use of several anesthetic agents. Herein, we present our anesthetic practice and the use of tramadol in a 75-year-old female patient who underwent pulmonary lobectomy under general anesthesia and was diagnosed with Brugada syndrome.
\end{abstract}

Keywords: Brugada syndrome, tramadol, arrhythmia, analgesia, general anesthesia, postoperative complications, cardiac conduction defect

\section{Introduction}

Brugada syndrome is a disorder characterized by sudden death due to ventricular fibrillation without structural heart failure. Although three types of this syndrome have been defined, it can be recognized when the T-segment elevation is followed by T-wave negativity or flattening. The initial symptom in $20 \%$ to $30 \%$ of patients is supraventricular tachycardia. However, the right bundle branch block may not be detected in some cases. ${ }^{1,2}$

Although it is considered that tramadol may cause Brugada syndrome by blocking the voltage-dependent sodium channels, there is only one case report published in the literature. ${ }^{3}$ Herein, we present our anesthetic practice and the use of tramadol in a 75-year-old female patient who underwent pulmonary lobectomy under general anesthesia and was diagnosed with Brugada syndrome, and discuss the safe use of tramadol in Brugada syndrome in light of literature data.

\section{Ethics approval and consent to participate}

Written informed consent was obtained for the publication of this case report and accompanying images from the patient who participated in this study.

\section{Case}

A 75-year-old female patient was admitted to the hospital with a squamous-cell tumor located in the right upper lobe of the lung. Her medical history included thyroidectomy due to multinodular goiter, Brugada syndrome, and penicillin and morphine allergy. She had arrhythmia and long QT syndrome 4 months prior and genetic testing showed p.K421fs*9 (c1265insA) (heterozygous) mutation in the KCNQ1 gene. She was diagnosed with Brugada syndrome. Echocardiography showed mild pulmonary hypertension (systolic blood pressure $30 \mathrm{mmHg}$ ), moderate pulmonary insufficiency, 1st-2nd
Correspondence: Cengiz Sahutoglu Ege Universitesi Tip Fakultesi, Anesteziyoloji ve Reanimasyon AD, 35100 , Bornova, Izmir, Turkey

Tel +902323902143

Fax +902323397687

Email csahutoglu@yahoo.com 
degree tricuspid regurgitation, and Stage 1 left ventricular diastolic dysfunction. Her heart rate was $58 / \mathrm{min}$ with sinus rhythm, and the QT interval was $44.5 \mathrm{~mm}$ on electrocardiography (ECG) (Figure 1). Complete blood count, biochemistry, and bleeding time test results were all within normal limits and the patient was on propafenone hydrochloride $300 \mathrm{mg}$ and levothyroxine sodium $25 \mu \mathrm{g}$.

Premedication with diazepam $5 \mathrm{mg}$ (orally) was administered one night before and on the morning of surgery. Written informed consent was obtained and she was taken to the operation room. She was monitored with ECG and pulse oximetry; and invasive arterial pressure, central venous pressure, body temperature, and urine flow were measured. Adhesive pads were applied to her back for defibrillation and an external defibrillator was available at the bedside. Anesthesia induction was performed with $5 \mathrm{mg} \mathrm{kg}^{-1}$ pentothal sodium, $2 \mu \mathrm{g}$ $\mathrm{kg}^{-1}$ fentanyl, and $0.6 \mathrm{mg} \mathrm{kg}^{-1}$ rocuronium bromide, while anesthesia was maintained with remifentanil infusion $\left(0.25^{-1}\right.$ $\mu \mathrm{g} \mathrm{kg}^{-1} \mathrm{~min}^{-1}$ ), desflurane (0.5-1 minimum alveolar concentration [MAC] $)$ and, if necessary, with rocuronium bromide. The patient was intubated with a double-lumen tube and was then laterally positioned. She underwent right upper lobectomy with left lateral position and right thoracotomy. The total duration of surgery was $300 \mathrm{~min}$ and no arrhythmias were observed during surgery. However, during the operation, hypotension $(60 / 40 \mathrm{mmHg})$ developed once, therefore, the dose of anesthetic agents was reduced and volume replacement and $5 \mathrm{mg}$ of ephedrine was administered. Following lobectomy, the first dose of tramadol infusion was applied in the operation room. For decurarization, the patient was intravenously injected with sugammadex sodium at a dose of $4 \mathrm{mg} \mathrm{kg}^{-1}$ and was extubated at $4 \mathrm{~min}$ in the operating room. For analgesia, tramadol infusion (400 mg daily) using the patient-controlled analgesia device and intramuscular injection of diclofenac sodium two times (b.i.d.) was initiated. The patient was monitored in the intensive care unit for $24 \mathrm{hrs}$ and started to receive oral tramadol 150 $\mathrm{mg}$ /day for analgesia in the ward. On the third postoperative day, subfebrile fever $\left(37.8^{\circ} \mathrm{C}\right)$ and atrial fibrillation (Figure 2) developed. She was referred to cardiology, and propafenone hydrochloride was discontinued. Then, intravenous administration of metoprolol $5 \mathrm{mg}$ was administered, and oral maintenance therapy with propranolol hydrochloride $40 \mathrm{mg}$ b.i.d. was initiated. The central catheter was withdrawn after the detection of Klebsiella pneumoniae and Escherichia coli in the swab sample of the catheter. Then, intravenous administration of amikacin $1000 \mathrm{mg}$ was initiated and treatment was completed on day 10. The patient was discharged from hospital on day 11 postoperatively. Written informed consent was obtained for the publication of this case report and accompanying images from the patient who participated in this study.

\section{Discussion}

Brugada syndrome was first reported in 1992 by Pedro Brugada. The mean age of onset of arrhythmias is 40 years, and more than $90 \%$ of patients are males. Approximately $25 \%$ of patients have a family history of syncope, ventricular

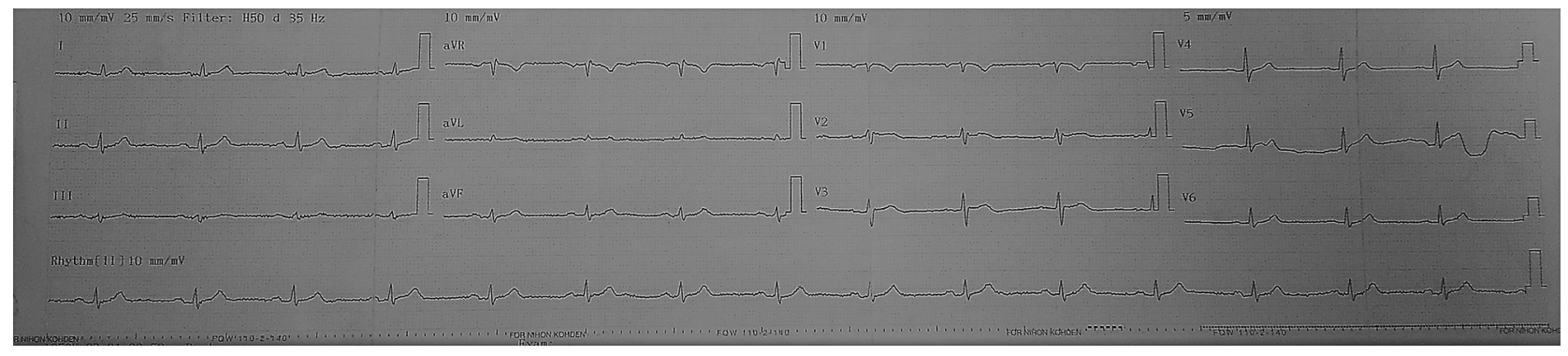

Figure I Preoperative electrocardiography of the patient.

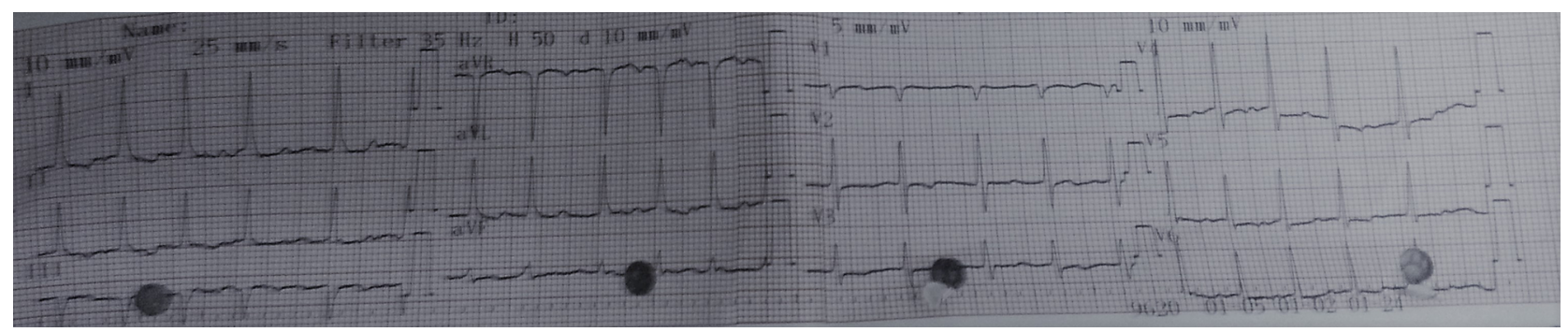

Figure 2 Atrial fibrillation on the third postoperative day (fever and infection). 
fibrillation, or suspected sudden cardiac death. ${ }^{1}$ On ECG, ST segment elevation and right bundle branch block pattern in precordial leads are mostly observed. In young adults without structural heart anomalies, this syndrome accounts for $50 \%$ of sudden deaths. It is difficult to diagnose Brugada syndrome and its genetic transition is autosomal dominant. This syndrome is also genetically associated with a gene mutation on chromosome 3 which encodes the human cardiac sodium channels. ${ }^{1,2}$

In our case, Brugada syndrome was diagnosed by genetic testing. There was no family history of sudden death at a young age and the sex of the patient was female. In addition, ST elevation and right bundle branch block were not evident in the precordial leads. In patients without a confirmed diagnosis, Brugada syndrome may go unnoticed according to such ECG findings, and arrhythmias may be induced with normal anesthesia protocols.

There is no established treatment protocol to prevent tachyarrhythmias in Brugada syndrome. Beta-blockers, bretylium, lidocaine, mexiletine, or magnesium, which were previously used for this purpose, failed; however, sotalol was shown to be effective in one case. ${ }^{4}$ In case of recurrent syncope, internal cardiac defibrillators (ICDs) should be placed. Cardioversion is the most optimal treatment option for intraoperative tachyarrhythmias. ${ }^{4}$ In our case, an ICD was not required. Rather, adhesive pads were applied to her back to intervene in case of tachyarrhythmia and the defibrillator was held in the working position in the room. Tachyarrhythmia (atrial fibrillation) which developed in the postoperative period was attributed to the presence of fever and infection, and was managed with metoprolol and propranolol hydrochloride.

Previous studies have shown that antiarrhythmic drugs (amiodarone, verapamil, propranolol, and lidocaine), local anesthetics (bupivacaine and lidocaine), propofol, metoclopramide, anticholinesterase agents (neostigmine and pyridostigmine), and anesthetics and analgesics (ketamine and tramadol) used in anesthesia practice have arrhythmogenic effects and may cause Brugada syndrome. Therefore, these agents should be avoided whenever possible (Class IIb recommendation level)..$^{3-6}$

In a study, Kloesel et $\mathrm{al}^{5}$ reported intraoperative arrhythmia in 17 anesthesia applications in eight patients. Twenty different ST alterations were reported in four patients. ST alterations developed after the administration of propofol infusion, lidocaine, fentanyl and remifentanil, oxymetazoline, granisetron, etomidate, and dexamethasone. However, no complications were seen. Therefore, the authors concluded that propofol and local anesthetics had a theoretical risk of arrhythmogenic potential in Brugada syndrome patients, although there was no clear evidence. ST-segment elevations can be seen particularly in patients receiving propofol infusion and attention should be paid to electrolyte disturbances such as hyperkalemia, hypokalemia, and hypercalcemia; and hyperthermia and bradycardia which may trigger arrhythmia. ${ }^{5}$ Duque et $\mathrm{al}^{7}$ reported that although hemodynamic complications occurred in Brugada syndrome using propofol, local anesthetics, tramadol, and metoclopramide, direct association between these complications and the use of these drugs was not observed. They stated that the clinical risk for Brugada syndrome was unknown at therapeutic doses of the drugs and that stronger clinical evidence was needed to prevent its use. However, they suggested that these medicines should be avoided in the presence of alternative medicines. In our case, no arrhythmias were observed during surgery. However, during the operation, hypotension $(60 / 40 \mathrm{mmHg})$ developed once, and the patient was treated by reducing the dose of anesthetic agents and administering volume replacement and $5 \mathrm{mg}$ of ephedrine.

Tramadol is a widely prescribed synthetic opioid analgesic and exhibits poor $\mu$ receptor agonist activity. In addition, it inhibits serotonin and norepinephrine reuptake. It has been shown that tramadol reduces amphibian action potential in the sciatic nerve and exerts a local anesthetic effect in humans by blocking sodium channels. Tachyarrhythmia including enlargement in the QRS and QT interval and ventricular fibrillation occurs, which resembles tricyclic antidepressant intoxication after its overuse ${ }^{8,9} \mathrm{Cole}$ et $\mathrm{al}^{3}$ found Brugada syndrome-like ECG findings in a 47-year-old male patient who received tramadol $60 \mathrm{mg}$ for a suicide attempt. In his initial ECG taken in the emergency department, sinus tachycardia, ST elevation in the V1-V3, and ECG findings similar to type 1 Brugada syndrome with the right bundle branch block were observed. However, he had no electrolyte disturbance or fever, and coronary occlusion was excluded through coronary angiography. In addition, serum tramadol levels were found to be extremely high and tramadol intoxication was diagnosed.

In an in vitro study, Haeseler et a ${ }^{6}$ investigated the effects of commonly used opioids on the voltage-dependent sodium channels. In their study, sufentanil, fentanyl, and tramadol slowly blocked the voltage-dependent $\mathrm{Na}^{+}$channels similar to meperidine, whereas morphine did not show such an effect. The authors concluded that there was no correlation between the blocking power and opioid potency. In our case, propofol and lidocaine were avoided for anesthesia induction. 
Although fentanyl was used for analgesia and remifentanil for anesthesia maintenance, no ECG alterations were observed. Although thoracic epidural block or paravertebral block is the gold standard for postoperative analgesia in patients with thoracotomy, the use of local anesthetics was limited by the presence of Brugada syndrome in our case. Fentanyl and especially remifentanil were not considered for postoperative pain management in our case, due to their short duration of effect and the risk of respiratory depression. In addition, as our patient suffered from morphine allergy, tramadol infusion was preferred due to the inadequate number of postoperative analgesics. In addition, attention was paid so that the dose of tramadol administered did not exceed $400 \mathrm{mg}$ per day. In our case, as it was an antidote of rocuronium as neuromuscularblocker rocuronium, sugammadex was used to antagonize the neuromuscular block. Therefore, the administration of an anticholinesterase was avoided.

\section{Conclusion}

Brugada syndrome is a rare rhythm disorder which may cause serious arrhythmias in patients undergoing anesthesia. Medications particularly used during local anesthesia, propofol infusion and anticholinesterases, can aggravate Brugada syndrome. These medicines should be avoided in the presence of alternative medicines. We believe that, when local anesthetics are avoided, morphine is a safe analgesic, whereas tramadol may be an option in patients with morphine contraindication. However, the best choice of analgesia in patients with Brugada syndrome will be clarified with future research involving larger case series.

\section{Author contributions}

Conception and design: CS, SK. Acquisition of data: CS, SK. Analysis and interpretation of data: CS, SK, FZA. Drafting the manuscript and revising it critically: CS, SK, FZA. All authors read the manuscript and have given their final approval of the version that is for publication.

\section{Disclosure}

The authors report no conflicts of interest in this work.

\section{References}

1. Berne P, Brugada J. Brugada syndrome 2012. Circ J. 2012;76(7): $1563-1571$.

2. Brugada J, Brugada P, Brugada R. The syndrome of right bundle branch block ST segment elevation in V1 to V3 and sudden death--the Brugada syndrome. Europace. 1999;1(3):156-166.

3. Cole JB, Sattiraju S, Bilden EF, Asinger RW, Bertog SC. Isolated tramadol overdose associated with Brugada ECG pattern. Pacing Clin Electrophysiol. 2012;35(8):e219-221.

4. Maury P, Hocini M, Haïssaguerre M. Electrical storms in Brugada syndrome: review of pharmacologic and ablative therapeutic options. Indian Pacing Electrophysiol J. 2005;5(1):25-34.

5. Kloesel B, Ackerman MJ, Sprung J, Narr BJ, Weingarten TN. Anesthetic management of patients with Brugada syndrome: a case series and literature review. Can J Anaesth. 2011;58(9):824-836.

6. Haeseler G, Foadi N, Ahrens J, Dengler R, Hecker H, Leuwer M. Tramadol, fentanyl and sufentanil but not morphine block voltageoperated sodium channels. Pain. 2006;126(1-3):234-244.

7. Duque M, Santos L, Ribeiro S, Catré D. Anesthesia and Brugada syndrome: a 12-year case series. J Clin Anesth. 2017;36:168-173.

8. Katsuki R, Fujita T, Koga A, Liu T, Nakatsuka T, Nakashima M, Kumamoto E. Tramadol, but not its major metabolite (mono-O-demethyl tramadol) depresses compound action potentials in frog sciatic nerves. Br J Pharmacol. 2006;149(3):319-327.

9. Altunkaya H, Ozer Y, Kargi E, Ozkocak I, Hosnuter M, Demirel CB, Babuccu $\mathrm{O}$. The postoperative analgesic effect of tramadol when used as subcutaneous local anesthetic. Anesth Analg. 2004;99(5):1461-1464.
Journal of Pain Research

\section{Publish your work in this journal}

The Journal of Pain Research is an international, peer reviewed, open access, online journal that welcomes laboratory and clinical findings in the fields of pain research and the prevention and management of pain. Original research, reviews, symposium reports, hypothesis formation and commentaries are all considered for publication.

\section{Dovepress}

The manuscript management system is completely online and includes a very quick and fair peer-review system, which is all easy to use. Visit http://www.dovepress.com/testimonials.php to read real quotes from published authors. 